\title{
Trapping of Atoms by the Counter-Propagating Stochastic Light Waves
}

\author{
V. I. Romanenko, L. P. Yatsenko \\ Institute of Physics of the National Academy of Sciences of Ukraine, 46, Nauky Ave., \\ Kyiv 03680, Ukraine
}

\begin{abstract}
We calculate the temperature of the atoms in the field of counter-propagating stochastic light waves (the chaotic-field model). We show that the temperature of the atomic ensemble depends on the autocorrelation time of the waves, their intensity and the detuning of the carrier frequency of the waves from the atomic transition frequency. The field can form a onedimensional trap for atoms, as is readily seen from our previous investigation of light-pressure force on an atom in counter-propagating stochastic light waves [V. I. Romanenko, B. W. Shore, L. P. Yatsenko, Opt. Commun. 268 (2006) 121-132]. We carry out numerical simulation of the atomic ensemble using paramaters appropriate for sodium atoms. Analyzing the known investigation of the light-pressure force on atoms and their motion in the counter-propagating polychromatic waves, we suggest an hypothesis that any polychromatic counter-propagating waves that have a discrete spectrum, or waves described by a stationary stochastic process, one of which repeats the other, can form a trap for atoms.
\end{abstract}

Keywords: optical trap for atoms, laser cooling, Monte Carlo wave-function method

PACS: 37.10.De, 37.10.Gh, 37.10.Vz

\footnotetext{
${ }^{*} \mathrm{~V}$. I. Romanenko

Email address: vr@iop.kiev.ua (V. I. Romanenko)
} 


\section{Introduction}

Optical traps for atoms in which, in addition to the confinement, the atoms are cooled, are widely used in experiments with cold atoms. For example, confinement and simultaneous cooling of atoms is realized in a widely used magneto-optical trap [1], wherein the atoms are subjected to laser radiation and a magnetic field. Recently, after a series of articles discussing the formation of a trap for atoms by the trains of counter-propagating light pulses, one of which repeats the other [2, 3, 4, 5, 6], it was shown that such a trap can also cool atoms, provided that the pulse parameters are properly chosen [7, 8, 9]. Another trap that simultaneously confines and cools atoms, but which is based on two collinear standing waves that can be treated as counter-propagating bichromatic light waves, was proposed in [10] and investigated by the authors in the recent paper [11]. The centers of both types of trap, the trap based on the counter-propagating light pulses and the trap based on the counter-propagating bichromatic waves, are situated at the point where the optical paths of the counter-propagating waves, produced by the same laser, are equal.

The idea of a trap formed by the counter-propagating trains of light pulses can be simply explained for the case of $\pi$-pulses [2]. Let's consider a two-level atom $A$ at a point where the pulses do not overlap (Figure 1). The atom interacts with a $\pi$-pulse $L$ traveling leftward toward the point $O$ where the pulses "collide". After a time delay shorter then the spontaneous emission time $\tau_{s p}$ it interacts with the $\pi$-pulse $R$ propagating in the opposite direction. The first pulse excites the atom and pushes it toward the point $O$ due to the absorption of a photon. The interaction with the second pulse leads to stimulated emission of the photon in the direction of its propagation, pushing the atom again toward point $O$. If the pulse repetition period is large $\left(T \gg \tau_{s p}\right)$, the atom always occupies the ground state before the interaction with a counter-propagating pair of pulses and a trapping force is provided. More detailed consideration shows that the trap can be formed even by pulses with an area much smaller than $\pi$ and the condition $T \gg \tau_{s p}$ is not obligatory [9]. It should be noted that the light-pressure force on the atoms in the field of counterpropagating bichromatic waves ("bichromatic force" [12]) can be qualitatively interpreted as an interaction with counterpropagating light pulses [10, 12]. Both the bichromatic force and the force in the field of the counter-propagating laser pulses is equal to zero at the point where the optical paths of the counter-propagating waves, produced 

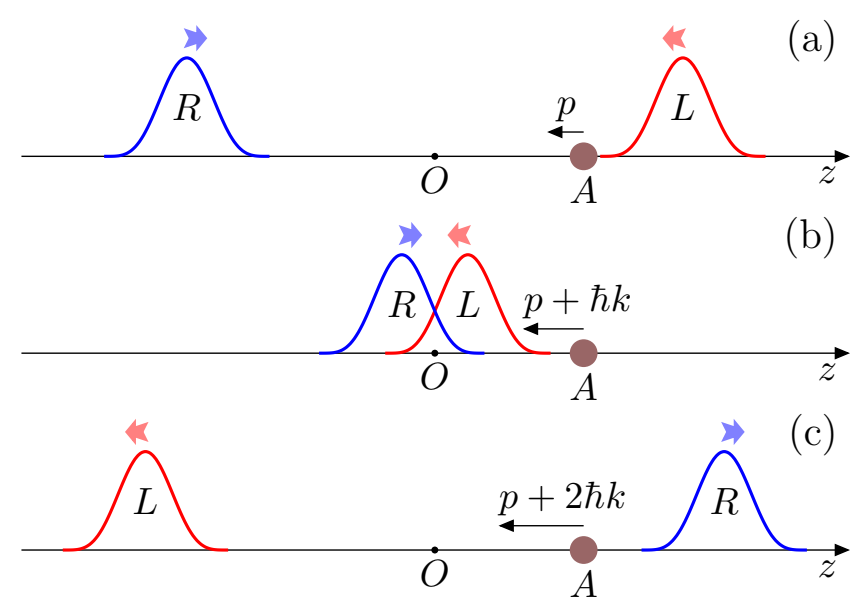

Figure 1: Interaction of a two-level atom (A) with counter-propagating $\pi$-pulses. (a) Initially the atom occupies the ground state and its momentum is $p$. (b) The pulse $L$, propagating in the negative $z$ direction, excites the atom and its momentum becomes $p+\hbar k$, where $\hbar k$ is the momentum of the photon. (c) Interaction with the counterpropagating pulse $R$ leads to the stimulated emission of a photon in the direction of $z$ axis and additional change of the atomic momentum by $\hbar k$. As a result, the atomic momentum is changed by $2 \hbar k$ after interaction with each pair of counter-propagating pulses. The momentum change is directed toward the point $O$ where the pulses "collide" 
by the same laser, are equal (the center of the trap). Near this point the light-pressure force depends almost linearly on the coordinate, thereby providing the restoring force for any deviation of an atom from the center of the trap. The force arises because the field strengths of the counter-propagating waves are correlated (in the opposite case the averaged force would be zero, as was shown in [13] for stochastic counter-propagating waves with time delay between waves more than the autocorrelation time). Therefore, we can expect the existence of the restoring force even in the case of the stochastic field, provided the autocorrelation time of the waves is much longer than the time of light propagation from the position of the atom to the center of the trap. This consideration is in good agreement with the calculation of the light-pressure force acting on an atom in counter-propagating stochastic waves [13].

The existence of the restoring force is a necessary but not sufficient condition for confinement of atoms in a trap. The laser radiation heats the atoms due to momentum diffusion from the scattering of laser photons. In optical traps for atoms the heating is compensated by a "friction force" which originates from the interaction of atoms with monochromatic standing waves that are slightly detuned from the atomic transition [1]. In the case of the counter-propagating stochastic wave we should determine whether the continuous spectrum of radiation with spectral width more then $1 / \tau_{s p}$ can cool atoms and how the spectral width affects the temperature of the possible cooling. In this paper we examine the interaction of atoms with counterpropagating stochastic plane waves (a chaotic-field model, where the real and imaginary parts of the complex amplitude of the electrical field fluctuate independently), and show that in this case, as in the case of the counterpropagating laser pulses or bichromatic field, the atoms can be confined and cooled by the same field. The trap under consideration combines the idea of the confinement of atoms by the counter-propagating waves and their cooling by white light [14, 15, 16] using the same laser radiation.

This paper is organized as follows. In Section 2 we present the trap model considered in this paper. In Section 3 the equations that describe the evolution of the atom in the stochastic field are written, in Section 4 the light-pressure force on an atom in the weak field is derived and the minimal temperature of the atomic ensemble in the stochastic field is found. In Section 5 we describe the numerical calculation routine used in the investigation. Results of the numerical calculations of the statistic properties of the ensemble of sodium atoms and their discussion are presented in Section 6. The 


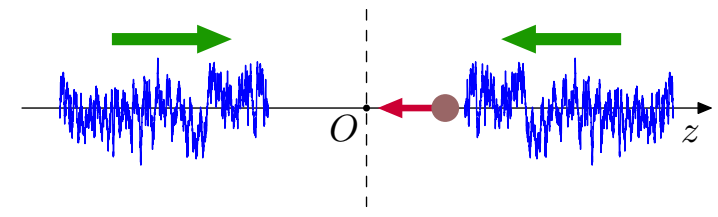

Figure 2: Schematic view of the trap. The atom (indicated by the circle) near the point $O$ is subjected to the field of the counter-propagating light waves with the stochastic envelope. The waves are produced by the same source and in the point $O$ their amplitudes and phases are equal. Due to the force of light pressure the atom moves near the center $O$ of the trap

conclusions are given in Section 7 . In the appendix, we explain the origin of the momentum diffusion of atoms in the field of laser radiation.

\section{Trap model}

According to the results of [13], the light pressure force in the field of the counter-propagating stochastic waves, one of which repeats the other with a certain time delay, is directed to the point where this delay is equal to zero. Thus, it is possible to form an optical trap by the stochastic light field. Whenever the radiation field of a multimode laser is close to stochastic [17, 18], it is possible to construct a trap on the basis of such lasers.

A schematic drawing of an one-dimensional trap for atoms, formed by the counter-propagating stochastic waves is depicted in Figure 2. The waves are produced by the same laser and are directed towards each other by a system of mirrors (not shown in the figure).

\section{Main equations}

In the calculations we assume the condition [19, 20]

$$
\frac{\hbar^{2} k^{2}}{2 m} \ll \hbar \gamma,
$$

which means that the light-pressure force is formed faster than the change of the atomic velocity will have a significant impact on its value (the heavy atom approximation). Here $k=\omega / c$ is the wave vector, $\omega$ is the carrier frequency of the waves, $\gamma$ is the rate constant of spontaneous emission. Criterion (1) is the basis for the semi-classical approach in derivation of the Doppler-cooling limit [20]. 
Let's consider an atom in the field of two counter-propagating waves, one of which repeats the other with some time delay. The origin of the coordinate system is situated at the point $O$ (see figure 2), where this delay is equal to zero. The atom with the coordinate $z$ is subjected to the electric field

$$
\mathbf{E}=\frac{1}{2} \mathbf{e}\left[E_{0}(t-z / c) \exp (i \omega t-i k z)+E_{0}(t+z / c) \exp (i \omega t+i k z)\right]+\text { c.c. }
$$

Here $\mathbf{e}$ is the unit vector of polarization.

We describe the laser radiation by the chaotic field model [17, 18], in which the real and imaginary parts of the complex amplitude of the field fluctuate independently. The ensemble average of the complex amplitude equals to zero and the autocorrelation functions of the real and the imaginary parts are

$$
\begin{aligned}
\left\langle\operatorname{Re} E_{0}(t) \operatorname{Re} E_{0}\left(t^{\prime}\right)\right\rangle & \left.=\frac{1}{2}\left|E_{0}\right|^{2} \exp -G\left|t-t^{\prime}\right|\right), \\
\left\langle\operatorname{Im} E_{0}(t) \operatorname{Im} E_{0}\left(t^{\prime}\right)\right\rangle & =\frac{1}{2}\left|E_{0}\right|^{2} \exp \left(-G\left|t-t^{\prime}\right|\right),
\end{aligned}
$$

where brackets $\langle>$ denote averaging over the ensemble of the possible realizations of the stochastic process, $G$ is the inverse correlation time and $\left|E_{0}\right|^{2}=\left\langle E_{0}(t) E_{0}^{*}(t)\right\rangle$ does not depend on time.

We use a simple two-level model of the atom-field interaction and assume the absence of polarization gradients. The difference of energies of the ground $|1\rangle$ and the excited $|2\rangle$ states is $\hbar \omega_{0}$. The light pressure force on the atom is given by the formula [1, 19]

$$
F=\left(\varrho_{12} \mathbf{d}_{21}+\varrho_{21} \mathbf{d}_{12}\right) \frac{\partial \mathbf{E}}{\partial z}
$$

where $\mathbf{d}_{12}$ and $\mathbf{d}_{21}$ are the matrix elements of the dipole moment, $\varrho_{12}$ and $\varrho_{21}$ are the non-diagonal elements of the density matrix $\varrho$.

According to (5) a force calculation requires we know not only the fields but also the density matrix of the atom. We use two approaches for calculating the density matrix of the atom. In our analytic calculation of the temperature of the atomic ensemble in a weak field we use the density matrix equation. In our numerical calculation of the statistical properties of the atomic ensemble we use the Monte Carlo wave-function method [21]. The results of both methods are compared in Section 6 and shows good agreement. 
Under the action of force (5) the atom accelerates according to Newton's law

$$
\dot{v}=F / m
$$

where $m$ is the mass of the atom and $v=\dot{z}$ is its velocity.

\section{Atoms in a weak field}

To explain laser cooling by a stochastic field, we will find the light pressure force (5) on an atom and the population of the excited state in a weak field $\left(\Omega_{0} \ll \gamma\right)$ using the equation for the density matrix

$$
i \hbar \frac{\partial}{\partial t} \varrho_{j k}=\sum_{l}\left(H_{j l} \varrho_{l k}-\varrho_{j l} H_{l k}\right)+i \hbar \sum_{l, m} \Gamma_{j k, l m} \varrho_{l m}
$$

where the Hamiltonian $H$ is equal to

$$
H=\hbar \omega_{0}|2\rangle\left\langle 2\left|-\mathbf{d}_{12}\right| 1\right\rangle\left\langle 2\left|\mathbf{E}(t)-\mathbf{d}_{21}\right| 2\right\rangle\langle 1| \mathbf{E}(t)
$$

The relaxation process in (7) is described by the term containing the array of values $\Gamma$. The non-zero elements of $\Gamma$ are

$$
\begin{aligned}
& \Gamma_{12,12}=\Gamma_{21,21}=-\gamma / 2 \\
& \Gamma_{11,22}=-\Gamma_{22,22}=\gamma
\end{aligned}
$$

After substitution of (8), (9) and (2) in (7), applying rotating wave approximation [22] and introducing the variables

$$
\sigma_{12}=\varrho_{12} e^{-i \omega_{0} t}, \quad \sigma_{21}=\varrho_{21} e^{i \omega_{0} t}
$$

we get

$$
\begin{aligned}
\frac{\partial}{\partial t} \varrho_{11}= & \frac{i}{2} \sigma_{12} e^{i \delta t}\left[\Omega_{1}^{*} e^{i k z}+\Omega_{2}^{*} e^{-i k z}\right]-\frac{i}{2} \sigma_{21} e^{-i \delta t}\left[\Omega_{1} e^{-i k z}+\Omega_{2} e^{i k z}\right] \\
& +\gamma \varrho_{22} \\
\frac{\partial}{\partial t} \sigma_{12}= & \frac{i}{2}\left(\varrho_{11}-\varrho_{22}\right) e^{-i \delta t}\left[\Omega_{1} e^{-i k z}+\Omega_{2} e^{i k z}\right]-\frac{\gamma}{2} \sigma_{12} \\
\sigma_{21}= & \sigma_{12}^{*}, \quad \varrho_{11}+\varrho_{22}=1
\end{aligned}
$$

where $\Omega_{1}=\Omega(t-z / c), \Omega_{2}=\Omega(t+z / c), \Omega(t)=-\mathbf{d}_{12} \mathbf{e} E_{0}(t) / \hbar, \delta=\omega_{0}-\omega$. The light-pressure force in this approximation is equal to 


$$
F=\hbar k \frac{i}{2} e^{i \delta t} \sigma_{12}\left[\Omega_{2}^{*} e^{-i k z}-\Omega_{1}^{*} e^{i k z}\right]-\hbar k \frac{i}{2} e^{-i \delta t} \sigma_{21}\left[\Omega_{2} e^{i k z}-\Omega_{1} e^{-i k z}\right] .
$$

We seek the quasi-stationary solution to the equations (11)-(13). This is achieved when $t \gg \gamma^{-1}$. Introducing the parameter $\varepsilon$ to denote the order of smallness of $\Omega_{1}, \Omega_{2}$ in comparison with $\gamma$ (after the end of the evaluation we put $\varepsilon=1$ ), we write the solution to (11)-(13) and the light-pressure force (14) in the form

$$
\begin{aligned}
\varrho_{11} & =\sum_{n=0}^{\infty} \varepsilon^{n} \varrho_{11}^{(n)}, \\
\sigma_{12} & =\sum_{n=0}^{\infty} \varepsilon^{n} \sigma_{12}^{(n)}, \\
F & =\sum_{n=0}^{\infty} \varepsilon^{n} f^{(n)} .
\end{aligned}
$$

From equations (11)-(13) it is easy to see that

$$
\begin{array}{r}
\varrho_{11}^{(0)}=1, \quad \varrho_{11}^{(1)}=0, \\
\sigma_{12}^{(0)}=\sigma_{12}^{(2)}=0, \quad f^{(0)}=f^{(1)}=0 .
\end{array}
$$

The first order solution to (11) $-(13)$ is

$$
\begin{aligned}
\sigma_{12}^{(1)}(t) & =\sigma_{12}^{(1)}(0) e^{-\frac{1}{2} \gamma t}+\frac{i}{2} \int_{0}^{t} e^{\frac{1}{2} \gamma\left(t^{\prime}-t\right)} \\
& \times e^{-i \delta t^{\prime}}\left[\Omega_{1}\left(t^{\prime}\right) e^{-i k z^{\prime}}+\Omega_{2}\left(t^{\prime}\right) e^{i k z^{\prime}}\right] d t^{\prime}, \\
\sigma_{21}^{(1)}(t) & =\sigma_{21}^{(1)}(0) e^{-\frac{1}{2} \gamma t}-\frac{i}{2} \int_{0}^{t} e^{\frac{1}{2} \gamma\left(t^{\prime}-t\right)} \\
& \times e^{i \delta t^{\prime}}\left[\Omega_{1}^{*}\left(t^{\prime}\right) e^{i k z^{\prime}}+\Omega_{2}^{*}\left(t^{\prime}\right) e^{-i k z^{\prime}}\right] d t^{\prime},
\end{aligned}
$$

where $z^{\prime}$ is the coordinate of the atom at time $t^{\prime}$. The second order term $f^{(2)}$ of the light-pressure force is

$$
\begin{aligned}
f^{(2)} & =-\frac{i}{2} \hbar k\left[\Omega_{1}^{*} \sigma_{12}^{(1)} e^{i \delta t+i k z}-\Omega_{1} \sigma_{21}^{(1)} e^{-i \delta t-i k z}\right] \\
& +\frac{i}{2} \hbar k\left[\Omega_{2}^{*} \sigma_{12}^{(1)} e^{i \delta t-i k z}-\Omega_{2} \sigma_{21}^{(1)} e^{-i \delta t+i k z}\right] .
\end{aligned}
$$


Substituting (20)-(21) in (22) and averaging force over the wavelength, we obtain the steady-state value of the light-pressure force

$$
\tilde{f}^{(2)}=\frac{-2 \hbar k \Omega_{0}^{2}\left(\frac{1}{2} \gamma+G\right) \delta k v}{\left[\left(\frac{1}{2} \gamma+G\right)^{2}+(\delta+k v)^{2}\right]\left[\left(\frac{1}{2} \gamma+G\right)^{2}+(\delta-k v)^{2}\right]} .
$$

Here we assumed $z=z_{0}+v t, z^{\prime}=z_{0}+v t^{\prime}$, where $v$ is the velocity of the atom, and took into account that, according to (3) and (41),

$$
\begin{aligned}
\left\langle\operatorname{Re} \Omega(t) \operatorname{Re} \Omega\left(t^{\prime}\right)\right\rangle & =\frac{1}{2} \Omega_{0}^{2} e^{-G\left|t-t^{\prime}\right|}, \\
\left\langle\operatorname{Im} \Omega(t) \operatorname{Im} \Omega\left(t^{\prime}\right)\right\rangle & =\frac{1}{2} \Omega_{0}^{2} e^{-G\left|t-t^{\prime}\right|},
\end{aligned}
$$

where $\Omega_{0}=\sqrt{\left\langle|\Omega|^{2}\right\rangle}$.

Equation (23) shows that the damping force occurs for $\delta>0$, when the carrier frequency $\omega$ of the the counter-propagating waves is less then the transition frequency $\omega_{0}$ in the atom. This corresponds with a well-known result for Doppler cooling of atoms by a monochromatic standing wave [1].

Similar calculations give the second-order solution to (11)-(13). When averaged over the wavelength, in the case of slow atoms $(k|v| \ll \gamma)$, this gives

$$
\tilde{\varrho}_{22}^{(2)}=\frac{\Omega_{0}^{2}\left(\frac{1}{2} \gamma+G\right)}{\gamma\left[\left(\frac{1}{2} \gamma+G\right)^{2}+\delta^{2}\right]} .
$$

Knowledge of the population of the excited state allows one to find the rate of spontaneous emission of photons $\gamma \tilde{\varrho}_{22}$, and, from this to calculate the momentum diffusion coefficient [23]

$$
D_{p}=\frac{1}{2}(\hbar k)^{2}(1+Q+\xi) \gamma \tilde{\varrho}_{22} .
$$

This coefficient quantifies the time dependence of the mean-squared momentum deviation $p$ from the mean value $\langle p\rangle$,

$$
\left\langle(p-\langle p\rangle)^{2}\right\rangle=2 D_{p} t
$$

In (27) the Mandel parameter $Q$ takes into account non-Poisson statistics of scattered photons. For low intensities this term is small and may be neglected. Parameter $\xi$ is determined by the angular distribution of the 
scattered photons. For an one-dimensional model considered here $\xi=1$ (photons are emitted in two opposite directions along the propagation of the light rays). It is for this model the well-known formula for the minimal temperature of the two-level atoms in the field of the standing wave,

$$
T_{D}=\frac{\hbar \gamma}{2 k_{B}}
$$

where $k_{B}$ is the Boltzmann constant, was derived.

Taking into account that the friction force, which slows the atom's velocity down, according to (23) is equal to

$$
\tilde{f}^{(2)}=-\alpha v
$$

for $v \ll \gamma / k$, where

$$
\alpha=\frac{2 \hbar k^{2} \Omega_{0}^{2}\left(\frac{1}{2} \gamma+G\right) \delta}{\left[\left(\frac{1}{2} \gamma+G\right)^{2}+\delta^{2}\right]^{2}},
$$

we calculate the temperature of the atomic ensemble [23]

$$
T=\frac{D_{p}}{\alpha k_{B}}=\frac{\hbar}{2 k_{B}}\left(G+\frac{\gamma}{2}\right)\left[\frac{G+\frac{\gamma}{2}}{\delta}+\frac{\delta}{G+\frac{\gamma}{2}}\right] .
$$

The temperature (32) reaches the minimum value

$$
T_{\text {min }}=\frac{D_{p}}{\alpha k_{B}}=\frac{\hbar}{k_{B}}\left(G+\frac{\gamma}{2}\right)
$$

when the detuning equals

$$
\delta=\delta_{\mathrm{opt}}=\left(G+\frac{\gamma}{2}\right) .
$$

Formula (33), when $G \rightarrow 0$, coincides with formula (29) for the minimal temperature of the atoms in the field of a standing monochromatic wave.

\section{Numerical calculation procedure}

The time evolution of the statistical properties of the atomic ensemble is calculated by the Monte Carlo wave-function method [21] with the subsequent ensemble averaging of the first and the second moments of the velocity and coordinate distribution functions. The state vector of the atom

$$
|\psi\rangle=c_{1}|1\rangle+c_{2} \exp \left(-i \omega_{0} t\right)|2\rangle,
$$


is determined from the Schrödinger equation

$$
i \hbar \frac{d}{d t}|\psi\rangle=H|\psi\rangle
$$

with the simulation of a quantum jump probability after every step of the integration.

The Hamiltonian in the Schrödinger equation (36) is

$$
H=H_{0}+H_{\text {int }}+H_{\text {rel }} \text {, }
$$

where

$$
H_{0}=\hbar \omega_{0}|2\rangle\langle 2|
$$

describes the atom in the absence of fields and relaxation. The term

$$
H_{\mathrm{int}}=-\mathbf{d}_{12}|1\rangle\left\langle 2\left|\mathbf{E}(t)-\mathbf{d}_{21}\right| 2\right\rangle\langle 1| \mathbf{E}(t),
$$

is responsible for the interaction of the atom with the field, and

$$
H_{\mathrm{rel}}=-\frac{i \hbar \gamma}{2}|2\rangle\langle 2|
$$

describes the relaxation due to the spontaneous emission. Because of this term the Hamiltonian (37), describing a closed two-level system, is nonHermitian, contrary to the Hamiltonian in the equation for the density matrix.

Substitution (35) and (37) in (36) gives the equation for $c_{1}$ and $c_{2}$. After applying the rotating wave approximation (RWA) [22] we finally arrive at

$$
\begin{aligned}
\frac{d}{d t} c_{1} & =-\frac{i}{2}\left[\Omega_{1} e^{-i k z}+\Omega_{2} e^{i k z}\right] c_{2} e^{-i \delta t} \\
\frac{d}{d t} c_{2} & =-\frac{i}{2}\left[\Omega_{1}^{*} e^{i k z}+\Omega_{2}^{*} e^{-i k z}\right] c_{1} e^{i \delta t}-\frac{\gamma}{2} c_{2} .
\end{aligned}
$$

We simulate the stochastic fields $\Omega_{1}, \Omega_{2}$ by an Ornstein-Uhlenbeck (OU) process $\Xi(t)$ [24] with the autocorrelation function

$$
\left\langle\Xi(t) \Xi\left(t^{\prime}\right)\right\rangle=B G e^{-G\left|t-t^{\prime}\right|} .
$$

This process is the solution to the Langevin differential equation

$$
\frac{d}{d t} \Xi(t)=-G \Xi(t)+G \xi(t) .
$$


Here $\xi(t)$ is the Gaussian white noise with the autocorrelation function

$$
\left\langle\xi\left(t_{1}\right) \xi\left(t_{2}\right)\right\rangle=2 B \delta\left(t_{1}-t_{2}\right)
$$

and $\delta(t)$ is the Dirac delta function.

The stochastic fields $\Omega_{1}(t), \Omega_{2}(t)$ are related to $\Xi(t)$ by the expressions

$$
\Omega_{1}(t)=\Xi(t-z / c), \quad \Omega_{2}(t)=\Xi(t+z / c) .
$$

The right-hand side of (43) coincides with the right-hand sides of (24), (25) for $\Omega_{0}=\sqrt{2 B G}$. Function $\Xi(t)$ is generated by the algorithm of Fox and others [25, 26], as was done in [13, 27, 28]. The value of $\Xi\left(t_{j+1}\right)$ at discrete times $t_{j}=t_{j-1}+\Delta t$ is calculated from $\Xi\left(t_{j}\right)$ according to

$$
\Xi\left(t_{j+1}\right)=\Xi\left(t_{j}\right) e^{-G \Delta t}+h\left(t_{j}\right) .
$$

Here $h\left(t_{j}\right)$ obeys Gaussian statistics with a zero first moment and the second moment given by

$$
\left\langle h\left(t_{j}\right)^{2}\right\rangle=B G\left(1-e^{-2 G \Delta t}\right) .
$$

The state vector $|\psi(t)\rangle$ is normalized to unity at time $t$. Because the Hamiltonian (37) is not Hermitian, after time step $\Delta t$ the state vector $\mid \psi^{(1)}(t+$ $\Delta t)\rangle$ is not normalized to unity. According to [21],

$$
\Delta P=1-\left\langle\psi^{(1)}(t+\Delta t) \mid \psi^{(1)}(t+\Delta t)\right\rangle
$$

is the probability of a quantum jump. If quantum jump occurs, the state vector becomes

$$
|\psi(t+\Delta t)\rangle=|1\rangle .
$$

In the opposite case we normalize the state vector and it becomes

$$
|\psi(t+\Delta t)\rangle=\frac{\left|\psi^{(1)}(t+\Delta t)\right\rangle}{\sqrt{1-\Delta P}} .
$$

To simulate a quantum jump, we introduce the random variable $\epsilon$, which is uniformly distributed between zero and one. If it is smaller than $\Delta P$, the quantum jump occurs.

The elements of the density matrix in (5) are expressed in terms of the probability amplitudes $c_{1}$ and $c_{2}$ by

$$
\varrho_{12}=c_{1} c_{2}^{*} e^{i \omega_{0} t}, \quad \varrho_{21}=c_{2} c_{1}^{*} e^{-i \omega_{0} t} .
$$


The light-pressure force (5) averaged over the period of the field's oscillations $2 \pi / \omega_{0}$ reads

$$
F=\hbar k \operatorname{Im}\left\{c_{1} c_{2}^{*} e^{i \delta t}\left[\Omega_{1}^{*} e^{i k z}-\Omega_{2}^{*} e^{-i k z}\right]\right\}\left(\left|c_{1}\right|^{2}+\left|c_{2}\right|^{2}\right)^{-1} .
$$

To simulate the atom's motion, we simultaneously solve equations (마) and (41), (42), where the light-pressure force is given by (53). In addition, the atomic momentum is stochastically changed due to the spontaneous emission and fluctuations of the absorption and the stimulated radiation emission process [19]. In our calculations, for the sake of simplicity, we accept that the atomic momentum changes by $\pm \hbar k$ with an identical probability in the course of spontaneous emission.

In the case of weak laser radiation intensity and negligible population of the excited state, the lightpressure force and the momentum diffusion coefficient are equal to the sums of corresponding quantities for each of the counter-propagating waves [29]. The spontaneous emission occurs after each absorption of a photon, and the fluctuations of the momentum change in the stimulated processes occur as often as the similar fluctuations in spontaneous radiation [19] (see Appendix). We applied this model to the computer simulation of the fluctuation-driven momentum variation in the process of the interaction of atoms with the field of the counter-propagating lowintensity pulses [9] and with the field of the counter-propagating bichromatic waves [11].

In the case of counter-propagating waves of high intensity, which is required to reduce the size of the atomic cloud in a trap, we may consider the momentum diffusion in the stimulated processes in a similar way, bearing in mind that the result should be treated as an estimation. A probable error can be associated with larger fluctuations of the momentum change owing to stimulated radiation processes in comparison with those occurring owing to the spontaneous radiation emission. The resulting calculation underestimates the atomic cloud size and the atomic temperature in the trap.

In summary, the calculation algorithm of the motion of atoms in the field is as follows. Equations (66) and (41), (41) are integrated by the fourthorder Runge-Kutta method of the fourth order. After every step we randomly decide whether a quantum jump occurs, and the state vector is renormalized. If the quantum jump occurs, the velocity of the atom changes by

$$
\Delta v=\hbar k\left(\epsilon_{1}-0.5\right) /\left(M\left|\epsilon_{1}-0.5\right|\right)+\hbar k\left(\epsilon_{2}-0.5\right) /\left(M\left|\epsilon_{2}-0.5\right|\right)
$$


where $\epsilon_{1,2}$ are random numbers uniformly distributed over the interval $[0,1]$. One of the terms simulates the momentum fluctuation due to a spontaneously emitted photon, and the other simulates the momentum fluctuation resulting from fluctuations of the stimulated absorption and emission of photons. The Ornstein-Uhlenbeck process, which describes the time dependence of the radiation field of the laser, is simulated by the equations (47) and (48).

After simulation of the motion of each atom in the ensemble we find the time dependence of the average velocity and the average coordinate of the atoms and the square root of the mean square deviation of the velocity and the coordinate from the corresponding average values.

\section{Results and discussion}

It is well known that the cycling atom-field interaction can be realized between two states of some atoms [1]. Our calculations was carried out for ${ }^{23} \mathrm{Na}$ atoms, in which the cyclic interaction can be realized for the transition $3^{2} S_{1 / 2}-3^{2} P_{3 / 2}$. The transition wavelength is $\lambda=589.16 \mathrm{~nm}$, the rate of spontaneous emission is $\gamma=2 \pi \times 10 \mathrm{MHz}$, the Doppler cooling temperature limit for sodium atoms is $T_{D}=240 \mu \mathrm{K}[1]$.

The motion of the atom in a stochastic field depends substantially on the field parameters. Among the whole set of parameters, we are interested in those that provide the motion of atoms in a narrow interval of coordinates $z$ at the vicinity of the trap center.

To test the method of calculation of the statistical parameters of the atomic ensemble we simulated the motion of sodium atoms in a weak field and compared the results with the analytical formula for the temperature of the atomic ensemble (32). Figure 3 shows a good agreement of numerical calculations of the temperature of the atomic ensemble with expression (32). Figure 4 shows an example of the time dependence of the average velocity and the standard deviation of the velocity from its mean value for the parameters corresponding to one of the points in Figure 3 ,

Over time, the size of the atomic cloud increases until the light pressure force prevents atoms from moving away from the center of the trap. Because the light-pressure force is small when the intensity of the laser beam is low, the atomic cloud does not stop expanding even for $t>100 \mathrm{~ms}$. Confinement of the atoms in the trap can be achieved by increasing the intensity of the laser radiation, as is shown in Figure 5. Atoms with the initial velocity $v_{0}=10 \mathrm{~m} / \mathrm{s}$ slow down during $0.35 \mathrm{~ms}$, and then their velocity fluctuates near 


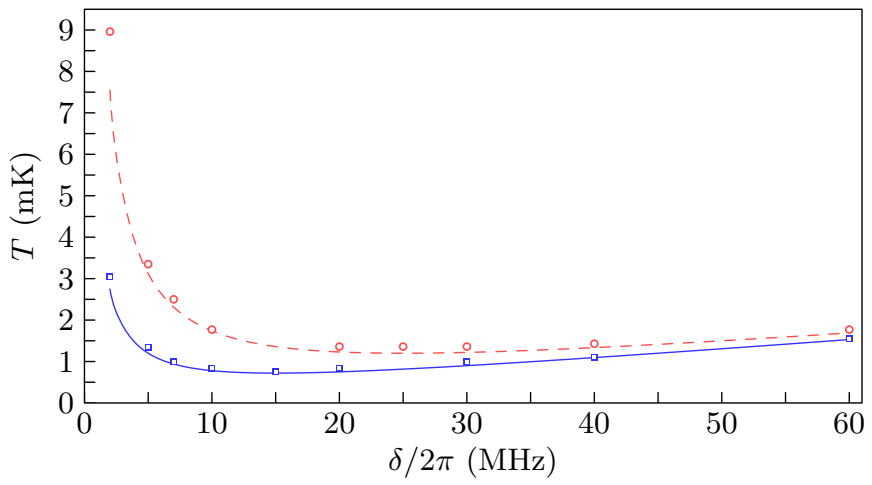

Figure 3: The dependence of the temperature of the atomic ensemble of 100 sodium atoms on the detuning $\delta$ of the carrier frequency of the stochastic waves $\omega$ from the atomic transition frequency $\omega_{0}$ for $\Omega_{0}=2 \pi \times 2 \mathrm{MHz}$ after $20 \mathrm{~ms}$ of the interaction of the atoms with the field. Squares and circles are the results of numerical calculation, lines show the calculation according to formula (32). Squares and solid curve correspond to $G=$ $2 \pi \times 10 \mathrm{MHz}, D=2 \pi \times 0.2 \mathrm{MHz}$, circles and dashed line correspond to $G=2 \pi \times 20 \mathrm{MHz}$, $B=2 \pi \times 0.1 \mathrm{MHz}$

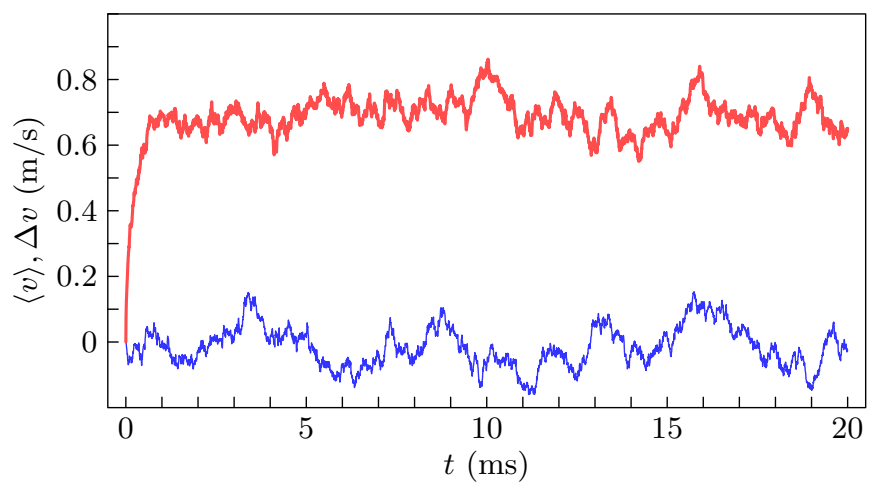

Figure 4: The time dependence of the average velocity $\langle v\rangle$ (thin line) and the standard deviation of the velocity from its average value $\Delta v$ (thick line) of the atomic ensemble of 100 sodium atoms for $\Omega_{0}=2 \pi \times 2 \mathrm{MHz}, \delta=2 \pi \times 20 \mathrm{MHz}, G=2 \pi \times 20 \mathrm{MHz}$, $B=2 \pi \times 0.1 \mathrm{MHz}$, initial velocity $v_{0}=0$ 

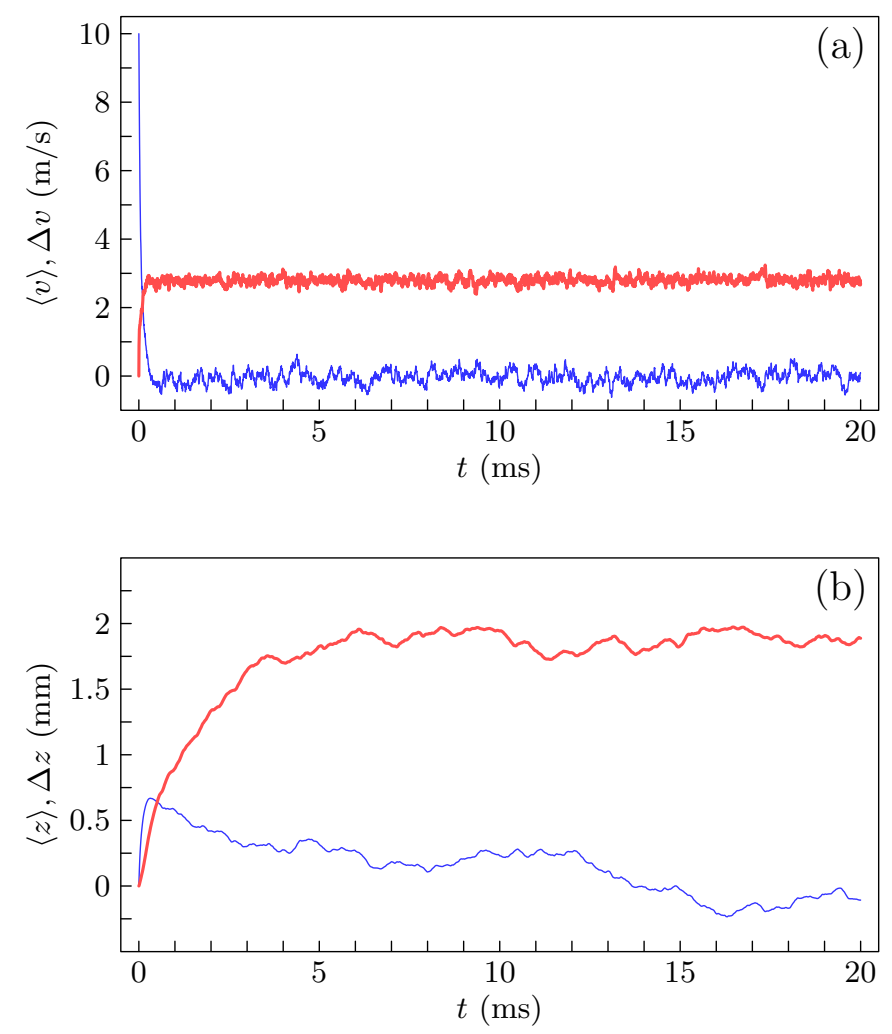

Figure 5: (a) The time dependence of the average velocity $\langle v\rangle$ (thin line) and the standard deviation of the velocity from the average value $\Delta v$ (thick line) for the atomic ensemble. (b) The time dependence of the average coordinate $\langle z\rangle$ (thin line) and the standard deviation of the coordinate from the average value $\Delta z$ (thick line) for the same atomic ensemble. Parameters: $\Omega_{0}=2 \pi \times 50 \mathrm{MHz}, \delta=2 \pi \times 15 \mathrm{MHz}, G=2 \pi \times 20 \mathrm{MHz}, B=2 \pi \times 62.5 \mathrm{MHz}$. Calculation was carried out for 200 sodium atoms, initial velocity $v_{0}=10 \mathrm{~m} / \mathrm{s}$ 
zero with the variance of $\Delta v=2.8 \mathrm{~m} / \mathrm{s}$, which corresponds to temperature $T=0.021 \mathrm{~K}$ (see Figure $5(\mathrm{a})$ ). The quasi-stationary distribution of atoms in space with mean square deviation from the the center of the trap $\Delta z=2 \mathrm{~mm}$ is reached after $6 \mathrm{~ms}$. The center of mass of the ensemble slows down and then tends to the origin of the coordinates (the center of the trap), where it fluctuates (see Figure 5(b)).

When the detuning $\delta$ becomes small, the friction force becomes less, and the decaying movement to the center of the trap changes to oscillation with the amplitude gradually decreasing to zero. An example of such a movement is shown in Figure 6. The root mean square value of the velocity at low detunings grows, resulting in rising temperature of the atomic ensemble, which is $0.15 \mathrm{~K}$ for Figure 6. Reduced friction force also explains approximately five times more deviation of $\langle z\rangle$ from the center of the trap at the beginning of the movement of atoms in Figure 6(b) compared to Figure 5(b).

Figure 7 shows the dependence of the temperature $T$ and the standard deviation $\Delta z$ of the sodium atoms from the center of the trap on the detuning $\delta$ for high intensity of the laser radiation $\left(\Omega_{0} \gg \gamma\right)$. As can be seen, the minimal temperature and the size of the atomic cloud is achieved when $\delta=2 \pi \times 15 \mathrm{MHz}$, which is significantly below $2 \pi \times 25 \mathrm{MHz}$ given by the formula (34). The minimal temperatures of the atomic ensemble for $\Omega_{0}=2 \pi \times 20 \mathrm{MHz}$ and $\Omega_{0}=2 \pi \times 50 \mathrm{MHz}$ are $7.1 \mathrm{mK}$ and $21.7 \mathrm{mK}$. These significantly exceed by $1.2 \mathrm{mK}$ the value given by formula (33). The temperature of sodium atoms and the size of the atomic cloud $(2 \Delta z)$ in the trap considered here are significantly greater (approximately 30 and 12 times) than corresponding values for Doppler cooling and trapping of sodium atoms in the widely used magneto-optical trap [30]. Nevertheless, our results show that even highly disordered field of the counter-propagating stochastic waves can form a trap for atoms. We believe that the physical nature of this trap is a correlation of the counter-propagating wave with the time dependent envelope, which is significant for distances less then $c / G$ from the center of the trap. Taking into account the possible formation of the trap by the trains of counter-propagating pulses [9] and the bichromatic counter-propagating waves [11], we suggest a hypothesis that any polychromatic counter-propagating waves with discrete spectrum or waves described by a stationary stochastic process, one of which repeats the other, can form a trap for atoms. 

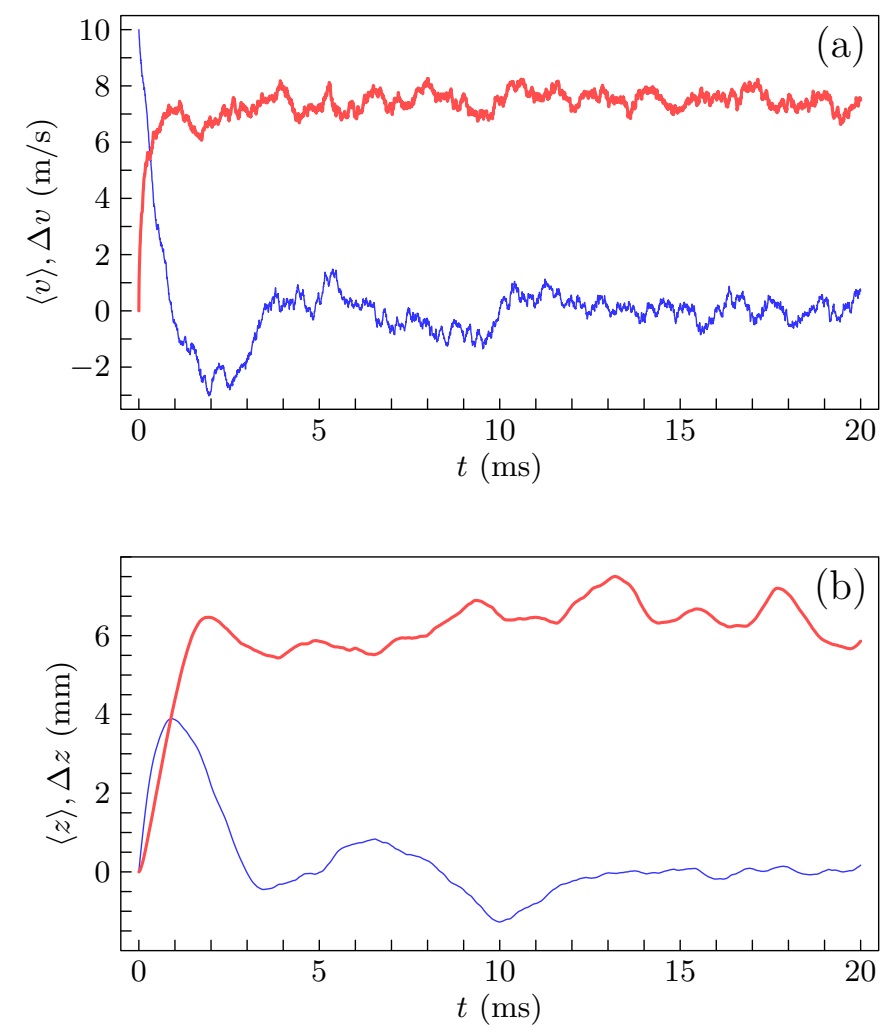

Figure 6: (a) The time dependence of the average velocity $\langle v\rangle$ (thin line) and the standard deviation of the velocity from the average value $\Delta v$ (thick line) for an atomic ensemble. (b) The time dependence of the average coordinate $\langle z\rangle$ (thin line) and the standard deviation of the coordinate from the average value $\Delta z$ (thick line) for the same atomic ensemble. Parameters: $\Omega_{0}=2 \pi \times 50 \mathrm{MHz}, \delta=2 \pi \times 1 \mathrm{MHz}, G=2 \pi \times 20 \mathrm{MHz}, B=2 \pi \times 62.5 \mathrm{MHz}$. Calculation was carried out for 200 sodium atoms, initial velocity $v_{0}=10 \mathrm{~m} / \mathrm{s}$ 

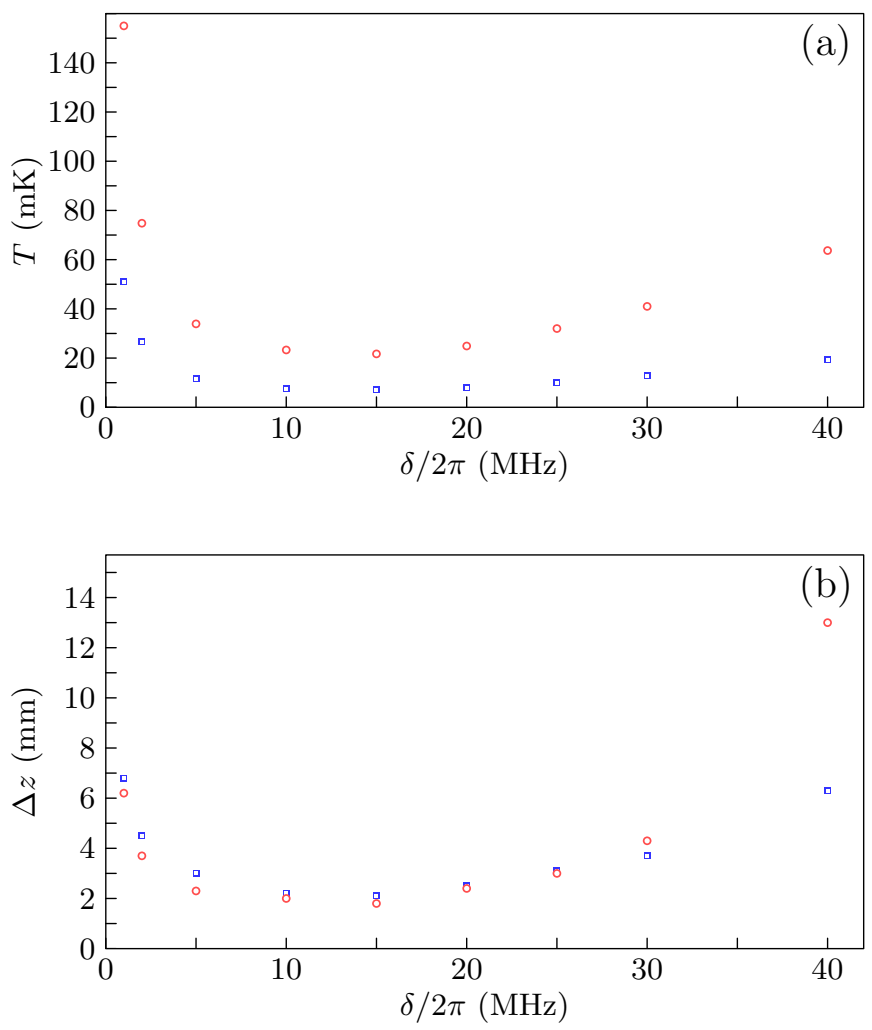

Figure 7: The dependence of (a) the temperature $T$ of the atomic ensemble and (b) the standard deviation $\Delta z$ of sodium atoms from the center of the trap on the detuning $\delta$. Parameters: $G=2 \pi \times 20 \mathrm{MHz}$, squares $-\Omega_{0}=2 \pi \times 20 \mathrm{MHz}, B=2 \pi \times 10 \mathrm{MHz}$, circles $-\Omega_{0}=2 \pi \times 50 \mathrm{MHz}, B=2 \pi \times 62.5 \mathrm{MHz}$. Calculation was carried out for 200 sodium atoms 


\section{Conclusions}

The simulation of the motion of atoms in the field of counter-propagating stochastic waves (the chaotic-field model), one of which repeats the other, shows that the atoms can be confined in a small area (a few $\mathrm{mm}$ ) near the point where the amplitudes and phases of the counter-propagating waves are the same. The minimal temperature of the atoms in low-intensity counterpropagating waves is determined by the rate of spontaneous emission of photons by the atoms in the excited state and the autocorrelation time of the stochastic process. If the intensity of the waves is large, it significantly affects the temperature of the atoms.

We believe that the trap formed the stochastic waves and the trap formed by the counter-propagating pulses or bichromatic waves have a common physical basis, the correlation of the counter-propagating waves. This point of view leads to the suggestion that any polychromatic counter-propagating waves with discrete spectrum or waves described by a stationary stochastic process, one of which repeats the other, can form a trap for atoms.

Because the temperature of the atomic ensemble in low-intensity counterpropagating stochastic waves is determined by the width of the spectrum of the stochastic field and the rate of spontaneous emission, such fields can be used for the formation of ensembles of atoms with a given (higher than Doppler cooling limit) temperature.

\section{Acknowledgements}

Funding: This work was supported by the State Fund for Fundamental Research of Ukraine [grant number F64/26-2016]; National Academy of Sciences of Ukraine in the frame of the goal-oriented complex program of fundamental researches "Fundamental issues in creation of new nanomaterials and nanotechnologies" [grant number 3/16-H). We thank B. W. Shore for careful reading of the manuscript.

\section{Appendix A. Momentum diffusion of atoms in the field of one travelling wave}

Here we explain the origin of the momentum diffusion of atoms in the field of laser radiation for an example of one travelling wave. We follow the book [19], adapting the presentation for one-dimensional model considering here. 
We assume that the atom's momentum at the time instant $t$ is $\mathbf{p}_{0}$. After time $\Delta t$ it becomes

$$
\mathbf{p}=\mathbf{p}_{0}+\hbar \mathbf{k}\left(N_{+}-N_{-}\right)-\sum_{s} \hbar \mathbf{k}_{s} .
$$

The second term describes the change of momentum due absorption and stimulated emission. The wave vector $\mathbf{k}$ is directed along $z$-axis, $N_{+}$and $N_{-}$are the numbers of the absorbed and emitted photons. The third term in (A.1) gives the momentum change due to the spontaneous emission of the photons with the wave vectors $\mathbf{k}_{s}$. For the one-dimensional model we assume $\mathbf{k}_{s}= \pm \mathbf{k}$ with equal probability and summation is over all the spontaneously emitted photons during time $\Delta t$.

After averaging (A.1) over an ensemble of atoms we find

$$
\langle\mathbf{p}\rangle=\left\langle\mathbf{p}_{0}\right\rangle+\hbar \mathbf{k}\left(\left\langle N_{+}\right\rangle-\left\langle N_{-}\right\rangle\right),
$$

where $\left\langle\mathbf{p}_{0}\right\rangle$ is the initial average momentum, $\left\langle N_{+}\right\rangle$is the average number of the absorbed photons, $\left\langle N_{-}\right\rangle$is the average number of the photon emitted due to stimulated emission. Here we took into account that

$$
\left\langle\sum_{s} \mathbf{k}_{s}\right\rangle=0
$$

Subtracting (A.1) from (A.2) we find the momentum fluctuation,

$$
\Delta \mathbf{p}=\mathbf{p}-\langle\mathbf{p}\rangle=\left(\mathbf{p}-\left\langle\mathbf{p}_{0}\right\rangle\right)+\hbar \mathbf{k} \Delta N_{i}-\sum_{s} \hbar \mathbf{k}_{s}
$$

where $\Delta N_{i}=N_{i}-\left\langle N_{i}\right\rangle$ is the variation of the difference $N_{i}=N_{+}-N_{-}$from the ensemble average value.

The average square of the momentum fluctuations along $z$-axis is

$$
\left\langle\Delta p_{z}^{2}\right\rangle=\left\langle\Delta p_{0 z}^{2}\right\rangle+\hbar^{2} k^{2}\left\langle\left(\Delta N_{i}\right)^{2}\right\rangle+\hbar^{2} k^{2}\left\langle N_{s}\right\rangle .
$$

Here $\left\langle N_{s}\right\rangle$ is the average number of the spontaneously emitted photons $\left\langle\Delta p_{0 z}^{2}\right\rangle$ is the initial momentum spreading, $\hbar^{2} k^{2}\left\langle\left(\Delta N_{i}\right)^{2}\right\rangle$ arises because of the stimulated processes (absorption and emission), $\hbar^{2} k^{2}\left\langle N_{s}\right\rangle$ is due to spontaneous emission.

Assuming the Poisson photons statistics, we find

$$
\left\langle\left(\Delta N_{i}\right)^{2}\right\rangle=\left\langle N_{i}\right\rangle
$$


Noting that $\left\langle N_{i}\right\rangle=\left\langle N_{s}\right\rangle$, we finally get

$$
\left\langle\Delta p_{z}^{2}\right\rangle=\left\langle\Delta p_{0 z}^{2}\right\rangle+\hbar^{2} k^{2}\left\langle N_{s}\right\rangle+\hbar^{2} k^{2}\left\langle N_{s}\right\rangle
$$

This equation can be interpreted physically as changing of atomic momentum by $\pm \hbar k$ due to spontaneous emission and by $\pm \hbar k$ due to stimulated process (emission an absorption) every spontaneous emission event.

\section{References}

[1] H. J. Metcalf, P. van der Stratten, Laser Cooling and Trapping, Springer-Verlag: New York, Berlin, Heidelberg, 1999.

[2] T. G. M. Freegarde, J. Waltz, W. Hänsch, Confinement and manipulation of atoms using short laser pulses, Opt. Commun. 117 (1995) $262-267$.

[3] A. Goepfert, I. Bloch, D. Haubrich, F. Lison, R. Schütze, R. Wynands, D. Meschede, Stimulated focusing and deflection of an atomic beam using picosecond laser pulses, Phys. Rev. A 56 (1997) R3354-R3357.

[4] V. I. Romanenko, L. P. Yatsenko, Theory of one-dimensional trapping of atoms by counterpropagating short pulse trains, J. Phys. B 44 (2011) 115305 .

[5] V. I. Balykin, Motion of an atom under the effect of femtosecond laser pulses: From chaos to spatial localization, JETP Lett. 81 (2005) 209213.

[6] D. N. Yanyshev, V. I. Balykin, Y. V. Vladimirova, V. N. Zadkov, Dynamics of atoms in a femtosecond optical dipole trap, Phys. Rev. A 87 (2013) 033411.

[7] V. I. Romanenko, A. V. Romanenko, Ye. G. Udovitskaya, L. P. Yatsenko, Momentum diffusion of atoms and nanoparticles in an optical trap formed by sequences of counter-propagating light pulses, Ukr. J. Phys. 58 (2013) 438-449.

[8] V. I. Romanenko, A. V. Romanenko, Ye. G. Udovitskaya, L. P. Yatsenko, Laser control of atomic and molecular motion by sequences of counterpropagating light pulses, J. Mod. Opt. 61 (2014) 839-844. 
[9] V. I. Romanenko, Ye. G. Udovitskaya, A. V. Romanenko, L. P. Yatsenko, Cooling and trapping of atoms and molecules by counterpropagating pulse trains, Phys. Rev. A 90 (2014) 053421.

[10] V. S. Voŭtsekhovich, M. V. Danileı̌ko, A. N. Negriı̌ko, V. I. Romanenko, L. Yatsenko, Observation of a stimulated radiation pressure of amplitude-modulated light on atoms, JETP Lett. 49 (1989) 161-164.

[11] V. I. Romanenko, A. V. Romanenko, L. P. Yatsenko, An optical trap for atoms on the basis of counter-propagating bichromatic light waves, Ukr. J. Phys. 64 (2016) 309-324.

[12] J. Söding, R. Grimm, Y. B. Ovchinnikov, P. Bouyer, C. Salomon, Shortdistance atomic beam deceleration with a stimulated light force, Phys. Rev. Lett. 78 (1997) 1420-1423.

[13] V. I. Romanenko, B. W. Shore, L. P. Yatsenko, Forces exerted on atoms by stochastic laser fields, Opt. Commun. 268 (2006) 121-132.

[14] J. Hoffnagle, Proposal for continuous white-light cooling of an atomic beam, Opt. Lett. 13 (2) (1988) 102-104.

[15] S. E. Park, H. S. Lee, E. joo Shin, T. Y. Kwon, S. H. Yang, H. Cho, Generation of a slow and continuous cesium atomic beam for an atomic clock, J. Opt. Soc. Am. B 19 (11) (2002) 2595-2602.

[16] R. Glover, T. Bastin, Optical collimation of an atomic beam using a white light molasses, JOSA B 32 (5) (2015) B1-B10.

[17] A. T. Georges, P. Lambropoulos, Saturation and stark splitting of an atomic transition in a stochastic field, Phys. Rev. A 20 (1979) 991-1004.

[18] A. T. Georges, Resonance fluorescence in markovian stochastic fields, Phys. Rev. A 21 (1980) 2034-2049.

[19] V. G. Minogin, V. S. Letokhov, Laser Light Pressure on Atoms, Gordon and Breach: New York, 1987.

[20] J. Dalibard, Y. Castin, Laser cooling from the semi-classical to the quantum regime, in: T. W. Hänsch, M. Inguscio (Eds.), Frontiers in Laser Spectroscopy: Varenna on Lake Como, Villa Monastero, 23 June-3 July 1992, Vol. 120, North Holland, 1994, pp. 445-476. 
[21] K. Mølmer, Y. Castin, J. Dalibard, Monte Carlo wave-function method in quantum optics, J. Opt. Soc. Am. B 10 (1993) 524-538.

[22] B. W. Shore, The Theory of Coherent Atomic Excitation, Vol. 1, Wiley: New York, 1990.

[23] C. S. Adams, E. Riis, Laser cooling and trapping of neutral atoms, Prog. Quant. Electr. 21 (1997) 1-79.

[24] W. Horsthemke, R. Lefever, Noise-Induced Transitions, Springer-Verlag: Berlin Heidelberg, 1984.

[25] R. F. Fox, I. R. Gatland, R. Roy, G. Vemuri, Fast, accurate algorithm for numerical simulation of exponentially correlated colored noise, Phys. Rev. A 38 (1988) 5938-5940.

[26] G. Vemuri, R. Roy, Effect of injected field statistics on transient dynamics of an injection seeded laser, Opt. Commun. 77 (1990) 318-324.

[27] A. Kuhn, S. Schiemann, G. He, G. Coulston, W. Warren, K. Bergmann, Population transfer by stimulated raman scattering with delayed pulses using spectrally broad light, J. Chem. Phys. 96 (1992) 4215-4223.

[28] L. P. Yatsenko, V. I. Romanenko, B. W. Shore, K. Bergmann, Stimulated Raman adiabatic passage with partially coherent laser fields, Phys. Rev. A 65 (2002) 043409.

[29] K. Mølmer, Limits of doppler cooling in pulsed laser fields, Phys. Rev. Lett. 66 (1991) 2301-2304.

[30] E. L. Raab, M. Prentiss, A. Cable, S. Chu, D. E. Pritchard, Trapping of neutral sodium atoms with radiation pressure, Phys. Rev. Lett. 59 (1987) 2631-2634. 